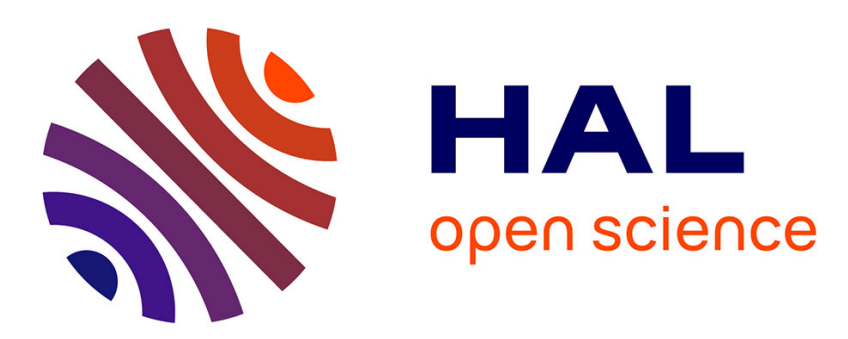

\title{
Effect of the Schmidt number on the diffusion of axisymmetric pancake vortices in a stratified fluid Ramiro Godoy-Diana, Jean-Marc Chomaz
}

\section{To cite this version:}

Ramiro Godoy-Diana, Jean-Marc Chomaz. Effect of the Schmidt number on the diffusion of axisymmetric pancake vortices in a stratified fluid. Physics of Fluids, 2003, 15 (4), pp.1058-1064. 10.1063/1.1558318. hal-01024932

HAL Id: hal-01024932

https://hal-polytechnique.archives-ouvertes.fr/hal-01024932

Submitted on 27 Aug 2014

HAL is a multi-disciplinary open access archive for the deposit and dissemination of scientific research documents, whether they are published or not. The documents may come from teaching and research institutions in France or abroad, or from public or private research centers.
L'archive ouverte pluridisciplinaire HAL, est destinée au dépôt et à la diffusion de documents scientifiques de niveau recherche, publiés ou non, émanant des établissements d'enseignement et de recherche français ou étrangers, des laboratoires publics ou privés. 


\section{AIP | Physics of Fluids}

\section{Effect of the Schmidt number on the diffusion of axisymmetric pancake vortices in a stratified fluid}

Ramiro Godoy-Diana and Jean-Marc Chomaz

Citation: Physics of Fluids (1994-present) 15, 1058 (2003); doi: 10.1063/1.1558318

View online: http://dx.doi.org/10.1063/1.1558318

View Table of Contents: http://scitation.aip.org/content/aip/journal/pof2/15/4?ver=pdfcov

Published by the AIP Publishing

\section{Articles you may be interested in}

Effect of the Prandtl number on a stratified turbulent wake

Phys. Fluids 22, 095102 (2010); 10.1063/1.3478841

Schmidt-number effects on the flow past a sphere moving vertically in a stratified diffusive fluid

Phys. Fluids 21, 026602 (2009); 10.1063/1.3075953

Evolution and instability of monopolar vortices in a stratified fluid

Phys. Fluids 15, 1033 (2003); 10.1063/1.1557528

Dipole formation by two interacting shielded monopoles in a stratified fluid

Phys. Fluids 14, 704 (2002); 10.1063/1.1431242

Dynamics of baroclinic vortices in a rotating, stratified fluid: A numerical study

Phys. Fluids 9, 419 (1997); 10.1063/1.869136

\section{AlP hameded}

Journal of Applied Physics is pleased to announce André Anders as its new Editor-in-Chief 


\title{
Effect of the Schmidt number on the diffusion of axisymmetric pancake vortices in a stratified fluid
}

\author{
Ramiro Godoy-Diana ${ }^{\text {a) }}$ and Jean-Marc Chomaz \\ LadHyX, CNRS-École Polytechnique, F-91128 Palaiseau Cedex, France
}

(Received 25 July 2002; accepted 15 January 2003; published 4 March 2003)

\begin{abstract}
An asymptotic analysis of the equations for quasi-two-dimensional flow in stratified fluids is conducted, leading to a model for the diffusion of pancake-like vortices in cyclostrophic balance. This analysis permits one to derive formally the model for the diffusion of an axisymmetric monopole proposed by Beckers et al. [J. Fluid Mech. 433, 1 (2001)], and to extend their results. The appropriate parameter for the perturbation analysis is identified as the square of the vertical Froude number $F_{v}=U / L_{v} N$, where $U$ is the horizontal velocity scale, $N$ is the Brunt-Väisälä frequency, and $L_{v}$ the vertical length scale. The physical mechanisms involved in the vortex decay are examined under the light of the asymptotic analysis results. In particular we discuss the effects of the Schmidt number, Sc, which measures the balance between the diffusion of momentum and the diffusion of the stratifying agent. Remarkably, the vertical transport due to the slow cyclostrophic adjustment is shown to slowdown the velocity decay when $\mathrm{Sc}$ is larger than unity whereas it accelerates it when Sc is smaller than unity. (C) 2003 American Institute of Physics.
\end{abstract}

[DOI: $10.1063 / 1.1558318$ ]

\section{INTRODUCTION}

Vortices in strongly stratified fluids exhibit some unique features due especially to the inhibition of vertical motions. One of the situations that has been identified, both in theory and experiments, is quasi-two-dimensional (Q2-D) flow for which the main motions are given in horizontal planes [see, e.g., Lin and Pao (1979); ${ }^{1}$ Riley et al. $\left.(1981)^{2}\right]$. A particular type of vortical structure with a clearly larger length scale in the horizontal direction than in the vertical one, the so-called pancake vortices, evolves in this regime [e.g., Spedding et al. (1996); ${ }^{3}$ Bonnier et al. (2000); ${ }^{4}$ Billant and Chomaz $\left.(2000)^{5}\right]$. The dynamical evolution of these vortices is in part determined by cyclostrophic balance, which is characterized by the equilibrium between the centrifugal force and the pressure gradient resulting from a deformation of the isopycnals inside the vortex.

An interesting model for the decay of a cyclostrophically balanced axisymmetric monopole has been proposed by Beckers, Verzicco, Clercx, and van Heijst (BVCH),${ }^{6}$ which provided a reference to be compared with experiments and numerical simulations. Although their model does well in representing the main behavior of the vortex, its heuristic character makes it difficult to explain the cases where it fails to reproduce all the features found in their numerical experiments. In this paper we make an asymptotic analysis of the equations for Q2-D stratified flow assuming the vertical Froude number $\left(F_{v}\right)$ small. At the lowest order of the expansion we get the model equations proposed by $\mathrm{BVCH}$ plus an equation for the evolution of the density perturbation. As we will discuss in the following, the advantage of obtaining it as a result of an asymptotic analysis relies on the proper esti-

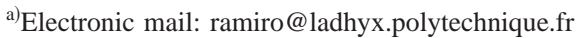

mations of the higher-order effects neglected in the model. Also, features depending on the ratio of momentum to stratifying agent diffusivities (Schmidt or Prandtl number for salt or temperature stratification, respectively) are analyzed, which allow us to gain insight into the processes governing the evolution of cyclostrophically balanced pancake vortices in real flows. In particular, we show that the secondary motion inside the vortex is reversed depending on whether $\mathrm{Sc}$ is smaller or larger than one. For $\mathrm{Sc}>1$ the secondary motion is dominated by the diffusion of momentum. Its observable effect is to slow down the decay of the horizontal velocity by transport and stretching of potential vorticity. On the contrary, when $\mathrm{Sc}<1$, the secondary motion is primarily driven by the density diffusion and it accelerates the damping of the velocity.

\section{Q2-D EQUATIONS}

Following Riley et al. ${ }^{2}$ and Lilly (1983), ${ }^{7}$ we describe the stably stratified system in terms of the density $(\widetilde{\rho})$ and pressure $(\tilde{p})$ perturbation fields with respect to a linear density profile $(\bar{\rho})$ and its corresponding hydrostatically balanced pressure field $(\bar{p})$. Thus, in a Cartesian system $\left(\mathbf{e}_{1} . \mathbf{e}_{2}, \mathbf{e}_{3}\right)$ with $\mathbf{e}_{3}$ opposing gravity and $\mathbf{x}=(x, y, z)$, the density and pressure fields can be written as

$$
\begin{aligned}
& \rho(\mathbf{x}, t)=\rho_{0}+\bar{\rho}(z)+\tilde{\rho}(\mathbf{x}, t), \\
& p(\mathbf{x}, t)=p_{0}+\bar{p}(z)+\tilde{p}(\mathbf{x}, t) .
\end{aligned}
$$

Defining the velocity field as $\mathbf{u}(\mathbf{x}, t)$, we can write the equations of motion for a stratified fluid in the Boussinesq approximation as

$$
\frac{D \mathbf{u}}{D t}=-\frac{1}{\rho_{0}} \nabla \tilde{p}-\frac{\tilde{\rho}}{\rho_{0}} g \mathbf{e}_{3}+\nu \nabla^{2} \mathbf{u},
$$




$$
\begin{aligned}
& \boldsymbol{\nabla} \cdot \mathbf{u}=0, \\
& \frac{D \tilde{\rho}}{D t}=\frac{\rho_{0} N^{2}}{g} u_{z}+\kappa \nabla^{2} \tilde{\rho},
\end{aligned}
$$

where $\nu=\mu / \rho_{0}$ is the mean kinematic viscosity, $N$ $=\left(-\left(g / \rho_{0}\right)(d \bar{\rho} / d z)\right)^{1 / 2}$ is the Brunt-Väisälä frequency, and $\kappa$ is the diffusivity of the stratifying agent (e.g., salt or temperature). Equation (5) for the evolution of the density perturbation was obtained from the transport equation of the stratifying agent (i.e., the temperature or the salinity) assuming that the density varies linearly with temperature or salinity. We define the scales $U, W, L_{h}$, and $L_{v}$ for the horizontal and vertical components of velocity $\mathbf{u}_{h}=\left(u_{x}, u_{y}\right), u_{z}$, and position $(x, y)$ and $z$, respectively. In addition, we note the existence of two relevant time scales: $T_{A}=L_{h} / U$, characterizing the evolution of horizontal advective motion, and $T_{N}$ $=N^{-1}$, a "buoyancy" time scale which is related to the internal gravity waves regime. The ratio of these two time scales defines a Froude number measuring the strength of inertial forces with respect to buoyancy forces, which we define as the horizontal Froude number:

$$
F_{h}=\frac{U}{L_{h} N} .
$$

Similarly we can define the vertical Froude number:

$$
F_{v}=\frac{U}{L_{v} N},
$$

which measures the ratio between the vertical length scale $L_{v}$ and the buoyancy length scale $L_{N}=U / N$. The latter can be interpreted as the maximum vertical displacement of a fluid parcel that converts all of its kinetic energy into potential energy [see, e.g., Tritton $(1988)^{8}$ ]. We note the evident relationship between these two Froude numbers $F_{h}=\alpha F_{v}$, which defines the aspect ratio $\alpha=L_{v} / L_{h}$.

Following Riley et al., ${ }^{2}$ we now proceed to a scaling analysis making some hypotheses on the dominant balances in the equations of motion (3)-(5) which aim at the description of the Q2-D regime and the pancake vortices. To begin with, as we are interested in motions that are far more important horizontally than vertically, we use the horizontal advection time scale $T_{A}=L_{h} / U$ to write the time derivatives in nondimensional form. We find the pressure scaling $\Pi$ $\sim \rho_{0} U^{2}$ by imposing the equilibrium of the pressure gradient and the advection term in the horizontal components of the momentum equation (3). Furthermore, from the balance between the pressure gradient and the buoyancy term in the vertical component of (3), the scale for the density perturbations can be written as $R \sim \rho_{0} U^{2} / g L_{v}$. Finally, to find the scale of the vertical velocity, we state that the partial time derivative of the density perturbation in Eq. (5) is balanced by the vertical velocity term, which renders $W \sim U F_{v} F_{h}$. This is equivalent to saying that variations in density are due to vertical displacement of fluid parcels. Using the previous scales and introducing the index $h$ to denote a vector in the horizontal plane (giving for example $D_{h} / D t=\partial / \partial t+\mathbf{u}_{h} \cdot \nabla_{h}$ for the horizontal material derivative), we obtain the following set of nondimensional equations:

$$
\begin{aligned}
& \frac{D_{h} \mathbf{u}_{h}}{D t}+F_{v}^{2} u_{z} \frac{\partial \mathbf{u}_{h}}{\partial z}=-\nabla_{h} \tilde{p}+\frac{1}{\operatorname{Re}}\left(\nabla_{h}^{2} \mathbf{u}_{h}+\frac{1}{\alpha^{2}} \frac{\partial^{2} \mathbf{u}_{h}}{\partial z^{2}}\right), \\
& \alpha^{2} F_{v}^{2}\left(\frac{D_{h} u_{z}}{D t}+F_{v}^{2} u_{z} \frac{\partial u_{z}}{\partial z}\right) \\
& =-\frac{\partial \tilde{p}}{\partial z}-\tilde{\rho}+\alpha^{2} \frac{F_{v}^{2}}{\operatorname{Re}}\left(\nabla_{h}^{2} u_{z}+\frac{1}{\alpha^{2}} \frac{\partial^{2} u_{z}}{\partial z^{2}}\right), \\
& \nabla_{h} \cdot \mathbf{u}_{h}+F_{v}^{2} \frac{\partial u_{z}}{\partial z}=0, \\
& \frac{D_{h} \tilde{\rho}}{D t}+F_{v}^{2} u_{z} \frac{\partial \tilde{\rho}}{\partial z}=u_{z}+\frac{1}{\operatorname{Re~Sc}}\left(\nabla_{h}^{2} \tilde{\boldsymbol{\rho}}+\frac{1}{\alpha^{2}} \frac{\partial^{2} \tilde{\rho}}{\partial z^{2}}\right),
\end{aligned}
$$

where we have used the definitions of the Reynolds $\mathrm{Re}$ $=U L_{h} / \nu$ and Schmidt (or Prandtl if temperature is the stratifying agent) $\mathrm{Sc}=\nu / \kappa$ numbers. Furthermore, if the vorticity vector is scaled by $U / L_{h}$, we obtain (using Cartesian coordinates to express the horizontal components)

$$
\begin{aligned}
& \omega_{z}=\nabla_{h} \times \mathbf{u}_{h}=\frac{\partial u_{x}}{\partial y}-\frac{\partial u_{y}}{\partial x}, \\
& \omega_{x}=\alpha F_{v}^{2} \frac{\partial u_{z}}{\partial y}-\frac{1}{\alpha} \frac{\partial u_{y}}{\partial z}, \\
& \omega_{y}=\frac{1}{\alpha} \frac{\partial u_{x}}{\partial z}-\alpha F_{v}^{2} \frac{\partial u_{z}}{\partial x} .
\end{aligned}
$$

The original Q2-D approximation proposed by Riley et al. ${ }^{2}$ considers both Froude numbers small $\left(F_{h} \ll 1\right.$ and $F_{v} \ll 1$ ), allowing one to develop all fields in powers of $F_{v}$ and obtaining at leading order the two-dimensional Euler equations for the horizontal components of the momentum equation with no vertical dependence.

\section{ASYMPTOTIC ANALYSIS}

In this section we develop an asymptotic analysis for the case of an axisymmetric monopole in order to formally derive a model for its decay. We consider the Q2-D approximation, where the horizontal fluid motions evolve under the advective time gauge $T_{A}$. Both Froude numbers are small $\left(F_{h} \ll 1\right.$ and $\left.F_{v} \ll 1\right)$ and we let the Reynolds number Re be of order $\alpha^{-2}$. This second hypothesis lets us find the terms associated with viscous diffusion at the lowest order of a perturbation analysis. When the condition on the vertical Froude number $F_{v} \ll 1$ is imposed, the condition on the horizontal Froude number $F_{h} \ll 1$ is satisfied even for $\alpha \leqslant 1$. In many cases involving slender vortices, however, the condition on the aspect ratio can be considered as $\alpha \ll 1$ and the diffusive terms in Eqs. (8)-(11) can be simplified by keeping only the vertical diffusion. In the present analysis we use the less restrictive hypothesis $\alpha \leqslant 1$ so that the full diffusion term appears at leading order. With the assumptions of both horizontal and vertical Froude numbers small, we are entitled to develop all fields in powers of $F_{v}$. Actually, as may be guessed from the quadratic dependence in $F_{v}$ of Eqs. (8)(11), it is convenient to consider powers of $F_{v}^{2}$, such that $\left(\mathbf{u}_{h}, u_{z}, \tilde{p}, \tilde{\boldsymbol{\rho}}\right)=\left(\mathbf{u}_{h 0}, u_{z 0}, \tilde{p}_{0}, \tilde{\boldsymbol{\rho}}_{0}\right)+F_{v}^{2}\left(\mathbf{u}_{h 2}, u_{z 2}, \tilde{p}_{2}, \tilde{\boldsymbol{\rho}}_{2}\right)+\cdots$. 
Using polar coordinates for the position $\mathbf{x}_{h}=(r, \theta)$ and the velocity $\mathbf{u}_{h}=\left(u_{r}, u_{\theta}\right)$, and assuming axisymmetric flow (i.e., independence of $\mathbf{u}, p$ and $\rho$ on $\theta$ ), the conservation of mass (10) implies at zeroth order that

$$
\frac{1}{r} \frac{\partial\left(r u_{r 0}\right)}{\partial r}=0,
$$

which gives

$$
u_{r 0}=0 \text {, }
$$

since $u_{r 0}$ vanishes at $r=0$ (i.e., there is no mass source at the vortex center). Moreover, from Eqs. (8), (9), and (11) the zeroth-order equations are simplified leading to the following system:

$$
\begin{aligned}
& -\frac{u_{\theta 0}^{2}}{r}=-\frac{\partial \tilde{p}_{0}}{\partial r}, \\
& \frac{\partial u_{\theta 0}}{\partial t}=\frac{1}{\operatorname{Re}}\left(\frac{\partial^{2} u_{\theta 0}}{\partial r^{2}}+\frac{1}{r} \frac{\partial u_{\theta 0}}{\partial r}-\frac{u_{\theta 0}}{r^{2}}+\frac{1}{\alpha^{2}} \frac{\partial^{2} u_{\theta 0}}{\partial z^{2}}\right), \\
& 0=-\frac{\partial \tilde{p}_{0}}{\partial z}-\tilde{\rho}_{0}, \\
& \frac{\partial \tilde{\rho}_{0}}{\partial t}=u_{z 0}+\frac{1}{\operatorname{Sc} \operatorname{Re}}\left(\frac{\partial^{2} \tilde{\rho}_{0}}{\partial r^{2}}+\frac{1}{r} \frac{\partial \tilde{\rho}_{0}}{\partial r}+\frac{1}{\alpha^{2}} \frac{\partial^{2} \tilde{\rho}_{0}}{\partial z^{2}}\right) .
\end{aligned}
$$

The radial (17) and vertical (19) momentum equations represent, respectively, cyclostrophic and hydrostatic balances. Together with Eq. (18), the asymptotic expansion recovers the heuristic diffusion model analyzed by BVCH. The model is extended since diffusion of density is now explicitly taken into account by Eq. (20), which relates density fluctuations and vertical velocity. Equation (18) is a closed equation and may be solved for any initial azimuthal velocity distribution $u_{\theta 0}(z, r)$. We will consider the self-similar solution used by $\mathrm{BVCH}$ in order to allow direct comparison of our results with their numerical simulations. It reads

$$
\begin{aligned}
u_{\theta 0}= & \frac{r}{2 \pi^{1 / 2}\left(2 \alpha^{2}+\frac{4}{\mathrm{Re}} t\right)^{1 / 2}\left(1+\frac{4}{\mathrm{Re}} t\right)^{2}} \\
& \times \exp \left(-\frac{\alpha^{2} z^{2}}{2 \alpha^{2}+\frac{4}{\mathrm{Re}} t}\right) \exp \left(-\frac{r^{2}}{1+\frac{4}{\mathrm{Re}} t}\right) .
\end{aligned}
$$

Now, from the equations for cyclostrophic and hydrostatic balances-Eqs. (17) and (19), respectively-the density field at leading order $\tilde{\rho}_{0}$ can be calculated, leading to

$$
\begin{aligned}
\tilde{\rho}_{0}= & \frac{-\alpha^{2} z}{4 \pi\left(2 \alpha^{2}+\frac{4}{\operatorname{Re}} t\right)^{2}\left(1+\frac{4}{\operatorname{Re}} t\right)^{3}} \\
& \times \exp \left(-\frac{2 \alpha^{2} z^{2}}{2 \alpha^{2}+\frac{4}{\operatorname{Re}} t}\right) \exp \left(-\frac{2 r^{2}}{1+\frac{4}{\operatorname{Re}} t}\right),
\end{aligned}
$$

which in turn lets us calculate the leading order vertical velocity $u_{z 0}$, using Eq. (20). Contour plots on the $(r, z)$ plane of these solutions are shown in Figs. 1(a)-1(c) for $\alpha=0.3$, $F_{v}=0.3, \mathrm{Re}=100$, and $\mathrm{Sc}=100$. The vertical and radial components of the nondimensional vorticity $\boldsymbol{\omega}$ also shown have been obtained in terms of the azimuthal velocity (21) as

$$
\omega_{z 0}=\frac{1}{r} \frac{\partial\left(r u_{\theta 0}\right)}{\partial r}, \quad \omega_{r 0}=-\frac{1}{\alpha} \frac{\partial u_{\theta 0}}{\partial z},
$$

whereas the azimuthal vorticity vanishes at leading order.

In order to find the radial velocity which appears to compensate the mass transported by $u_{z 0}$ we need to calculate the next order of the expansion since $u_{r 0}$ is zero. At order $F_{v}^{2}$ the conservation of mass gives

$$
\frac{\partial\left(r u_{r 2}\right)}{\partial r}+r \frac{\partial u_{z 0}}{\partial z}=0,
$$

which lets us calculate $u_{r 2}$. In Fig. 1(f) the contour plot of the initial distribution of $u_{r 2}$ corresponding to the case of Figs. 1(a)-1(e) is shown. The important radial extension of the $u_{r 2}$ field is a result of mass conservation. In the core of the vortex, Eq. (23) expresses that a converging (or diverging) vertical velocity plays the role of a source (or a sink) for the second-order radial flow. Outside of the vortex core the vertical velocity vanishes and the radial flow extends to infinity, decreasing only like $r^{-1}$ since $\partial\left(r u_{r 2}\right) / \partial r$ should be zero. In a real case the extent of the radial flow will be limited to a distance $r \sim F_{v}^{2}$ at which the next order dominates, and a matched asymptotic expansion would be required to find the far field. This is, however, beyond the scope of the present work.

With the expressions for the vertical and radial velocities, we can calculate also the azimuthal vorticity up to order $F_{v}^{2}$ as follows:

$$
\omega_{\theta}=F_{v}^{2}\left(\frac{1}{\alpha} \frac{\partial u_{r 2}}{\partial z}-\alpha \frac{\partial u_{z 0}}{\partial r}\right) .
$$

Azimuthal vorticity is an interesting quantity since it gives a snapshot of the secondary circulation in the $(r, z)$ plane, positive (negative) values of $\omega_{\theta}$ indicating clockwise (counterclockwise) motion. Azimuthal vorticity is represented in Fig. 2 for two cases. From the shape of the contours it can be inferred that the major contribution to its total value comes from the $\partial u_{r 2} / \partial z$ term in Eq. (24).

The effect of the secondary circulation advection on the mean azimuthal flow can be discussed using the $F_{v}^{2}$ order azimuthal momentum equation,

$$
\begin{aligned}
\frac{\partial u_{\theta 2}}{\partial t} & +u_{r 2} \frac{\partial u_{\theta 0}}{\partial r}+\frac{u_{\theta 0} u_{r 2}}{r}+u_{z 0} \frac{\partial u_{\theta 0}}{\partial z} \\
& =\frac{1}{\operatorname{Re}}\left(\frac{\partial^{2} u_{\theta 2}}{\partial r^{2}}+\frac{1}{r} \frac{\partial u_{\theta 2}}{\partial r}-\frac{u_{\theta 2}}{r^{2}}+\frac{1}{\alpha^{2}} \frac{\partial^{2} u_{\theta 2}}{\partial z^{2}}\right) .
\end{aligned}
$$

The term

$$
u_{r 2} \frac{\partial u_{\theta 0}}{\partial r}+\frac{u_{\theta 0} u_{r 2}}{r}+u_{z 0} \frac{\partial u_{\theta 0}}{\partial z}
$$



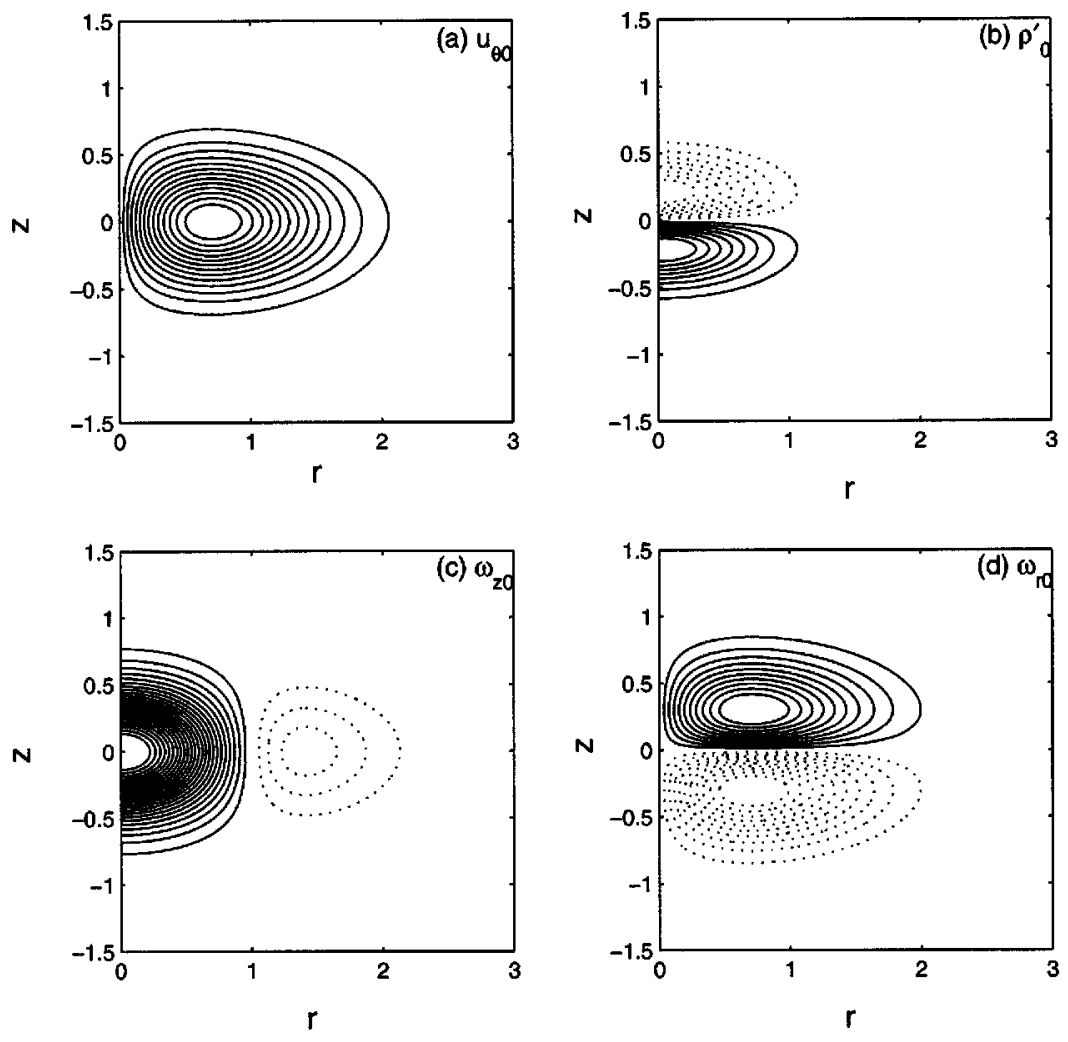

FIG. 1. Plots of (a) $u_{\theta 0}$, (b) $\tilde{\rho}_{0}$, (c) $\omega_{z 0}$, (d) $\omega_{r 0}$, (e) $u_{z 0}$, and (f) $u_{r 2}$ obtained from the diffusion model with $\alpha=0.3, \quad F_{v}=0.3, R e=100, \quad$ and $\mathrm{Sc}$ $=100$. The dotted contours represent negative values of the solid contours positive values. The positive contour intervals and increments are $\left(u_{\theta 0 \min }: \Delta u_{\theta 0}: u_{\theta 0 \max }\right)=(0.02: 0.02:$ $0.26), \quad\left(\tilde{\rho}_{0 \min }: \Delta \tilde{\rho}_{0}: \tilde{\rho}_{0 \max }=(0.01:\right.$ $0.01: 0.08), \quad\left(\omega_{z 0 \min }: \Delta \omega_{z 0}: \omega_{z 0 \max }\right)$ $=(0.05: 0.05: 1.2),\left(\omega_{r 0 \min }: \Delta \omega_{r 0}:\right.$ $\left.\omega_{r 0 \max }\right)=(0.015: 0.015: 0.15)$,

$\left(u_{z 0 \min }: \Delta u_{z 0}: u_{z 0 \max }\right)=(0.004: 0.004$ $: 0.04) \quad$ and $\quad\left(u_{r 2 \min }: \Delta u_{r 2}: u_{r 2 \max }\right)$ $=(0.002: 0.003: 0.029)$.
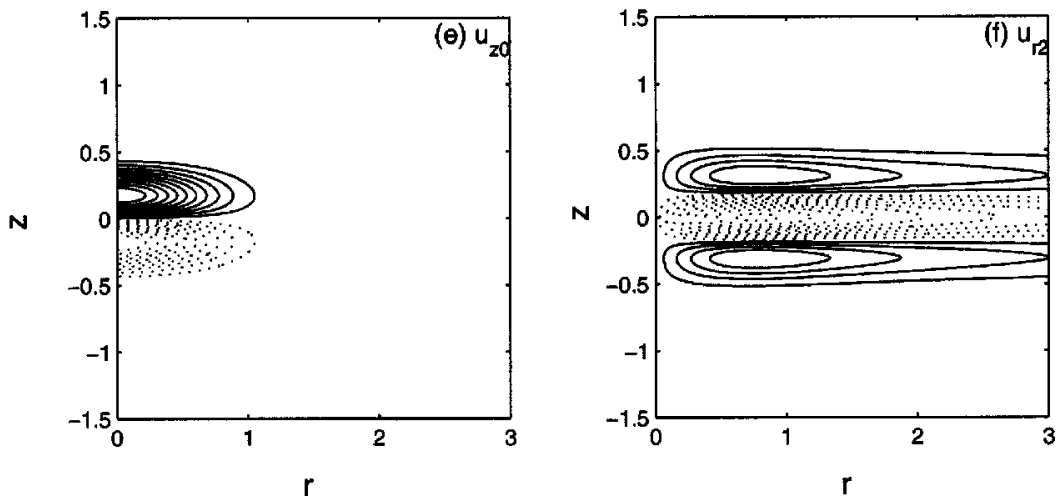

forces the $u_{\theta 2}$ field to depart from zero, while the right-handside of (25), representing the action of viscosity, acts the opposite way making $u_{\theta 2}$ relax back.

\section{SCHMIDT NUMBER EFFECTS ON THE VORTEX DECAY}

We have used the evolution equation for the density perturbation (20) to calculate the vertical velocity at zeroth order $u_{z 0}$. The dominant balance of terms in this equation, depends on the value of the Schmidt number Sc. For large $\mathrm{Sc}$, the diffusion of density is negligible and $u_{z 0}$ will be dominated by the $\partial \tilde{\rho}_{0} / \partial t$ contribution, which is driven only by the diffusion of horizontal momentum, i.e.,

$$
u_{z 0} \sim \frac{\partial \tilde{\rho}_{0}}{\partial t}=-\frac{\partial^{2}}{\partial t \partial z} \int \frac{u_{\theta 0}^{2}}{r} d r .
$$

The diffusion of momentum decreases the centrifugal force and the deflection of the isopycnals inside the vortex relaxes back to the horizontal. Since this relaxation may occur only by vertical transport, the induced vertical velocity should be positive above the vortex symmetry plane and negative below [Fig. 3(a)]. On the contrary, if Sc is small, the diffusion of density will represent the largest contribution to $u_{z 0}$, in the limit case eliminating the effect of $\partial \widetilde{\rho}_{0} / \partial t$, i.e.,

$$
u_{z 0} \sim-\frac{1}{\operatorname{Sc} \operatorname{Re}}\left(\frac{\partial^{2} \tilde{\rho}_{0}}{\partial r^{2}}+\frac{1}{r} \frac{\partial \tilde{\rho}_{0}}{\partial r}+\frac{1}{\alpha^{2}} \frac{\partial^{2} \tilde{\rho}_{0}}{\partial z^{2}}\right) .
$$

In this case the momentum diffusion has no or little role in determining the vertical velocity since its contribution is masked by the stratification diffusion, which acts on a faster time scale. Thus, the vertical transport should compensate the diffusion of density in order to maintain the cyclostrophic equilibrium. The induced vertical velocity is therefore negative above the vortex and positive below [Fig. 3(b)].

The resulting dynamics is opposite in each of these cases since, as mentioned earlier, the secondary circulation accompanying the vortex decay is driven by the vertical velocity $u_{z 0}$. An illustration of the two situations can be obtained 

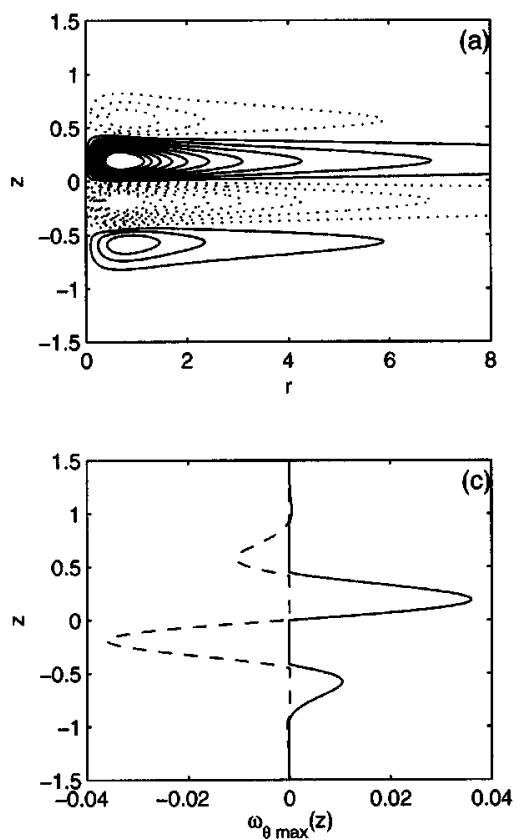
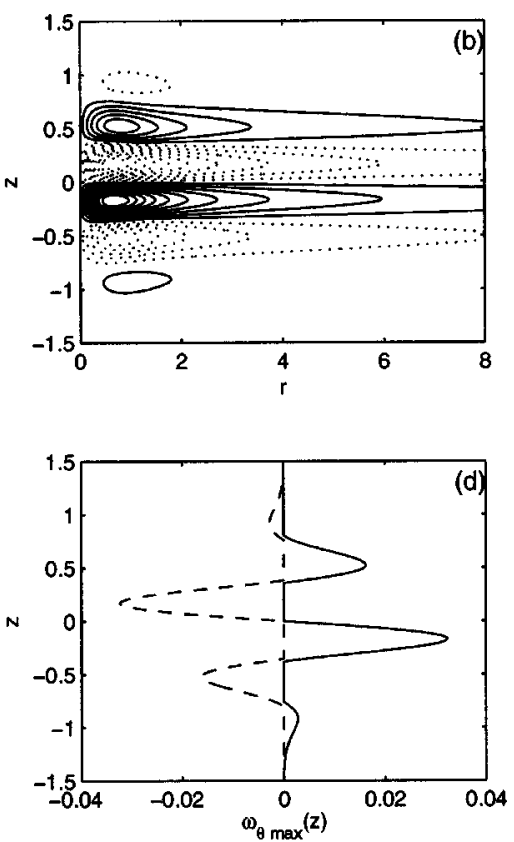

FIG. 2. Contour and extreme-value plots of $\omega_{\theta}$ at $t=0$ for $\alpha=0.4, F_{v}$ $=0.75, \operatorname{Re}=100,(\mathrm{a}),(\mathrm{c}) \mathrm{Sc}=700$, and (b), (d) $\mathrm{Sc}=0.7$. The dotted contours represent negative values of the solid contours positive values. The positive contour intervals and increments in (a) and (b) are $\left(\omega_{\theta \min }: \Delta \omega_{\theta}: \omega_{\theta \max }\right)$ $=(0.002: 0.003: 0.029)$. from expression (24) for $\omega_{\theta}$. In Fig. 4, plots of the maximum values of azimuthal vorticity [referred to as $\omega_{\theta \max }(t)$ ] are shown for various values of Sc, negative values of $\omega_{\theta \max }$ correspond to the cases turning counterclockwise on the upper $(r, z)$ plane. (We have kept the notation Sc of the Schmidt number for the whole range of values, even when for values smaller than 1 only the Prandtl number Pr could be realistic.) Contour plots of the initial values of $\omega_{\theta}$ for $\mathrm{Sc}=700$ and $\mathrm{Sc}=0.7$ were shown in Fig. 2. The main feature observed in Figs. 2 and 4 is the inversion on the rotation sense for the curves corresponding to Sc smaller than 1 . We explain this behavior as follows: for large Sc, the circulation pattern depicted by the $\omega_{\theta}$ contours represents a radial flow toward the vortex center and a vertical outward flow near the vortex axis. Following the idea from the previous paragraph, we remark that the vertical velocity $u_{z 0}$ driving this secondary circulation is only determined by the density distribution $\tilde{\rho}_{0}$ adjusting itself to the decaying $u_{\theta 0}$. For $\mathrm{Sc}<1$ the situation is reversed, since the stratifying agent diffuses faster than momentum and therefore the vertical velocity induced to preserve cyclostrophic balance, restoring the deformation of the isopycnals, points toward the vortex symmetry plane. Anticipating on the second order $u_{\theta 2}$ computation we may predict that the effect of the secondary circulation during the vortex decay when $\mathrm{Sc} \gg 1$ should retard the damping, since it will oppose the radial growth and the decay of vertical vor-
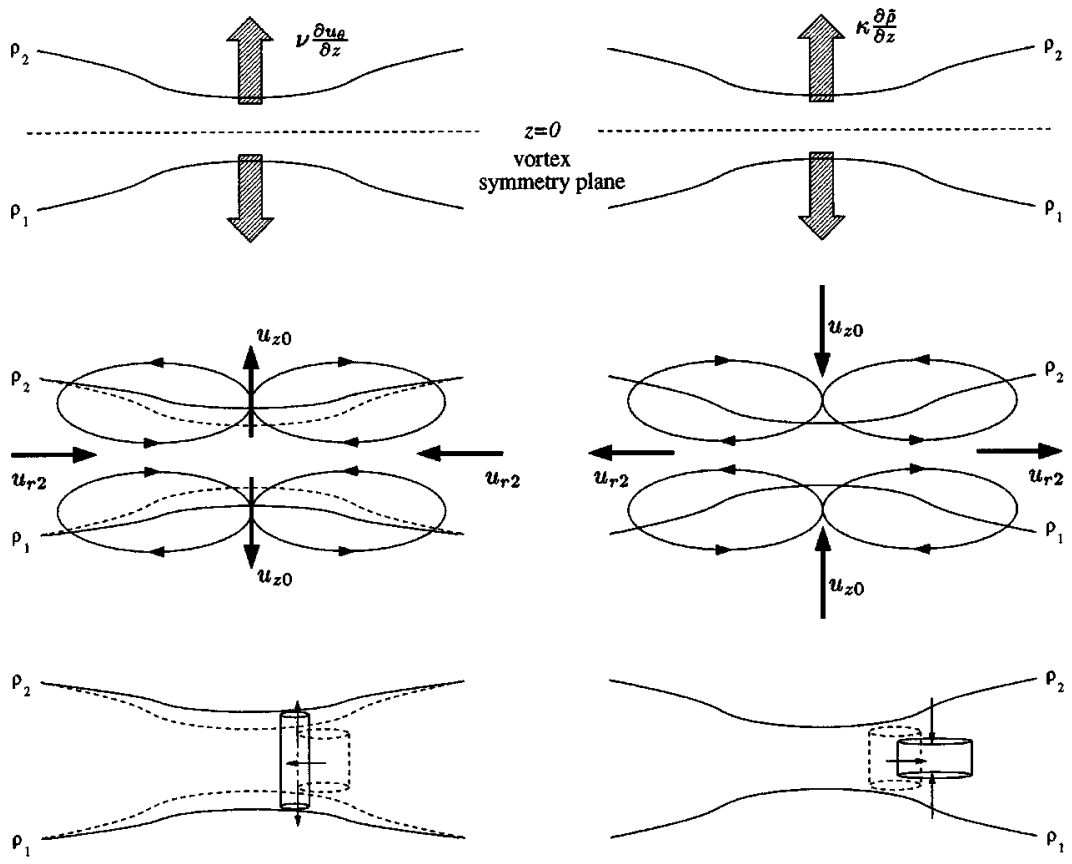

FIG. 3. Schematic diagrams of the secondary circulation during the vortex decay for (a) $\mathrm{Sc} \gg 1$ and (b) $\mathrm{Sc} \ll 1$. Isopycnals deflected toward the vortex symmetry plane are represented as solid lines. In the middle and bottom figures for case (a) an initial time is shown in dashed lines. The direction of the radial and vertical velocities is shown in the middle figures for both cases. Stretching (a) and squeezing (b) of vorticity is pictured schematically in the bottom figures (see the text for discussion). 


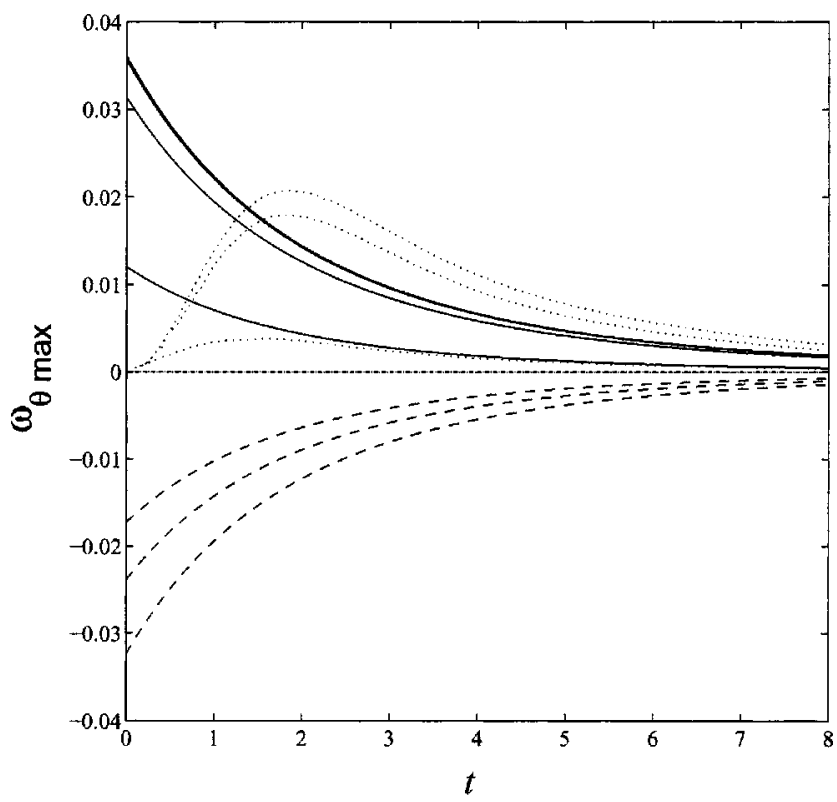

FIG. 4. Plots of $\omega_{\theta \max }(t)$ for $\alpha=0.4, F_{v}=0.75, \mathrm{Re}=100$. Asymptotic model results are shown in dashed and solid lines for (from bottom to top curve) $\mathrm{Sc}=0.7,0.8,0.9,1,10,100$, and 700. In dotted curves the results of the $\mathrm{BVCH}$ numerical simulations (see the text) for $\mathrm{Sc}=1,10$, and 100 (from bottom to top curve).

ticity by the radial transport and the stretching effect of vertical velocity, respectively. On the other hand, for $\mathrm{Sc}<1$ the situation is reversed, the outward flow being radial and the vertical inflow compressing the vortex. We expect this effect to enhance damping since the secondary circulation due to density diffusion will transport the vorticity radially outwards and produce a vortex compression effect. These features can be readily observed looking at the time evolution of the vertical vorticity $\omega_{z}$ and the vortex radius [defined as the value of $r=r^{\prime}$ which renders $\omega_{z}\left(r^{\prime}\right)=0$ ]. In order to do so, we need to calculate the $F_{v}^{2}$-order effect on the azimuthal velocity $u_{\theta 2}$, which requires solving Eq. (25). Since the transport terms in Eq. (25) are given in terms of the known quantities $u_{\theta 0}, u_{z 0}$, and $u_{r 2}$, the $u_{\theta 2}$ field can be computed numerically by solving a forced diffusion equation. However, in order to gain a better physical understanding of the role of the secondary circulation on the vortex evolution, we may estimate $u_{\theta 2}$ by neglecting the diffusion at this order and formally solving the simplified equation:

$$
\frac{\partial u_{\theta 2}}{\partial t}=-u_{r 2} \frac{\partial u_{\theta 0}}{\partial r}-\frac{u_{\theta 0} u_{r 2}}{r}-u_{z 0} \frac{\partial u_{\theta 0}}{\partial z} .
$$

This approximation is fully justified in the small Sc number limit. In other cases the $u_{\theta 2}$ thus computed is slightly overestimated but shows the proper trend. In Figs. 5 and 6 plots of the decaying maximum value of $\omega_{z}$ up to second order in $F_{v}^{2}$ and of the vortex radial growth are shown, respectively, for two different values of Sc. The radius $r^{\prime}$ is defined as the value of $r$ for which $\omega_{z}$ changes sign. Dashed lines in both figures represent the zeroth-order evolution; it is important to remember that at zeroth order azimuthal vorticity is almost zero, so the vortex decays exclusively as a result of diffusion of $u_{\theta}$-governed by Eq. (18). Over- and under-

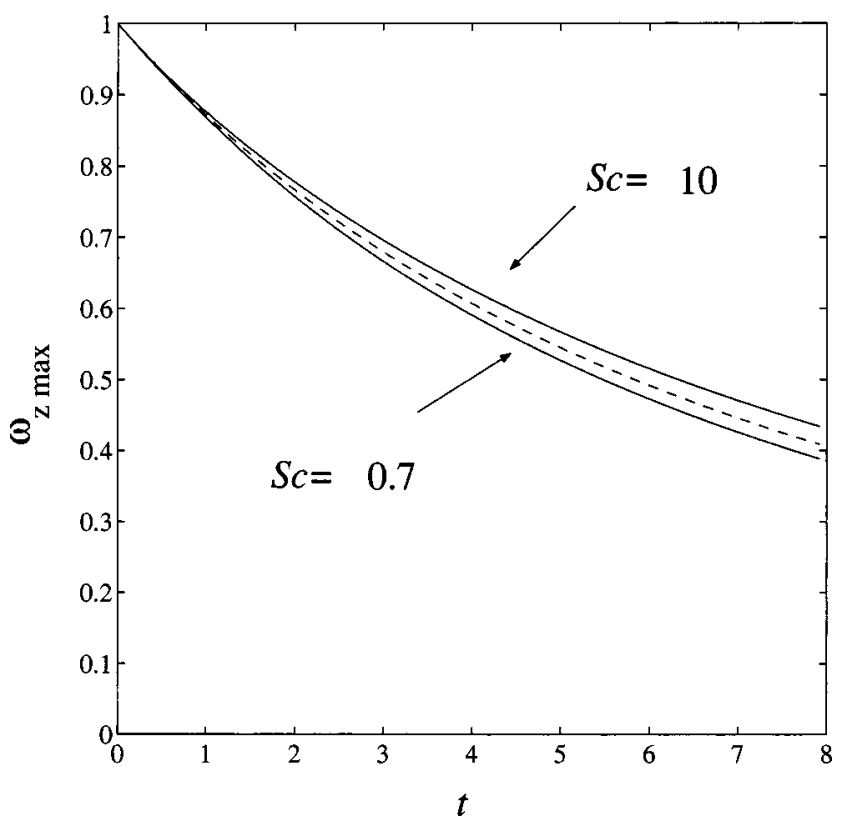

FIG. 5. Decay of $\omega_{z \max }(t)$. Zeroth order in dashed line and two cases of $F_{v}^{2}$ order in solid lines.

damped behaviors with respect to the zeroth-order values are clearly identified depending on the value of Sc. That is, for the case of $\mathrm{Sc}=10$, the $F_{v}^{2}$-order vortex damping, represented by the diminishing vertical vorticity and the growing radius, is slower than the zeroth-order prediction, while for the case of $\mathrm{Sc}=0.7$ the vortex decays faster due to the diffusion of the stratifying agent. For even smaller Sc, the diffusion is faster and occurs not on the viscous time scale $T_{\nu v}=L_{v}^{2} / \nu$ but on $T_{\nu v}$ Sc. This super-damping effect may be relevant in astro-

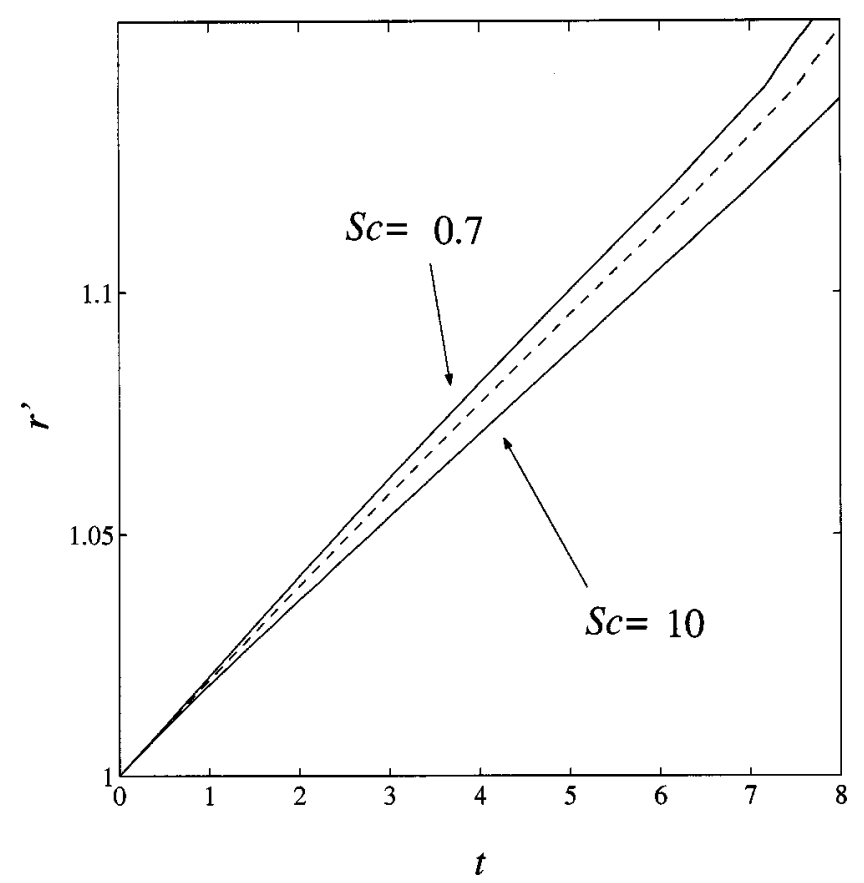

FIG. 6. Growth of $r^{\prime}(t)$. Zeroth order in dashed line and two cases of $F_{v}^{2}$ order in solid lines. 
physical situations where, for example, a metallic liquid is thermally stratified (as eventually in the Jovian core).

\section{COMPARISON WITH THE RESULTS OF BVCH}

In this section we compare the results of the asymptotic model with the numerical simulations of BVCH. We address again the decay of the secondary circulation on the $(r, z)$ plane represented by $\omega_{\theta \max }$ (Fig. 4). The numerical results of $\mathrm{BVCH}$ are plotted in dotted lines for $\alpha=0.4, F_{v}=0.75$, Re $=100$, and (from bottom to top) $\mathrm{Sc}=1,10,100$. The reason for the difference in the initial values is that, as detailed by $\mathrm{BVCH}$, their numerical simulations start with cyclostrophically balanced azimuthal velocity and density distribution, but with zero azimuthal vorticity. The first stage of the evolution shown is thus the increase of azimuthal vorticity to achieve the cyclostrophically balanced value. This stage corresponds to what has been referred to as cyclostrophic adjustment (see Ref. 6) and, as we can see in Fig. 4, it is superposed to the vortex decay for $t \leqslant 2$. The picture is rather different for the results of our asymptotic model: since the internal circulation corresponding to cyclostrophic balance is intrinsically included on the initial conditions, a nonzero initial value for $\omega_{\theta \max }$ is observed and there is no need for adjustment. In the later stages of the vortex evolution, the results of the asymptotic model are qualitatively consistent with the numerical simulations of $\mathrm{BVCH}$, and even in rather good quantitative agreement for the case of $\mathrm{Sc}=1$. It is noteworthy that the vertical Froude number value in the numerical simulations of BVCH reported here is 0.75 , which is out of the scope of the present asymptotic analysis valid for $F_{v}$ $\ll 1$. It is striking, however, that the asymptotic calculus succeeds in capturing the order of magnitude and the main trend of the vortex evolution, validating the physical interpretations about the diffusion process.

\section{CONCLUSIONS}

The diffusion of vortices in a stratified fluid has been discussed using an asymptotic expansion of the Q2-D equations of motion. The appropriate parameter for the expansion has first been identified as the squared vertical Froude number $F_{v}^{2}$. The zeroth-order equations yield an extended version of the diffusion model proposed by BVCH in Ref. 6 and at first order in $F_{v}^{2}$ the terms governing the departure from this model have been calculated. Of particular interest are two opposite effects associated with cyclostrophic balance which depend on the ratio of the diffusivities of momentum and stratifying agent, the Schmidt or Prandtl number, which has been referred to univocally as Sc. For high values of Sc, the stretching process resulting from cyclostrophic balance has been shown to slow down the vortex decay, acting against diffusive damping. A process of this kind may be invoked to explain the existence of long-lived vortical structures in nature (e.g., the meddies, see Ref. 9). Also, pancake vortices in laboratory experiments (where the usual setups are based on salt-stratified water tanks and $\mathrm{Sc} \cong 700$ ) certainly evolve under these effects. The opposite picture is found for Sc smaller than 1 (and in particular for $\mathrm{Sc}=0.7$ ), where the density diffuses faster than momentum and the secondary circulation driven by cyclostrophic balance induces a compression of the vortex, enhancing its diffusion. This over-damped situation occurs in atmospheric flows where $\mathrm{Sc}=0.7$ and it may explain why pancake turbulence is difficult to observe in thermally stratified gas experiments. The predictions of the asymptotic model are compared with the numerical simulations of BVCH and, even when the cases suitable for comparison are well beyond the $F_{v} \ll 1$ limit imposed by the asymptotic expansion, the main behavior of the vortex is successfully described.

\section{ACKNOWLEDGMENTS}

This work has benefited from useful discussions with Paul Billant. R.G.D. gratefully acknowledges support from CONACyT-México.

${ }^{1}$ J. Lin and Y. Pao, "Wakes in stratified fluids: A review," Annu. Rev. Fluid Mech. 11, 317 (1979).

${ }^{2}$ J. Riley, R. Metcalfe, and M. Weissman, "Direct numerical simulations of homogeneous turbulence in density stratified fluids," in Proceedings of the AIP Conference on Nonlinear Properties of Internal Waves, edited by B. West, La Jolla Institute, 1981, pp. 79-112.

${ }^{3}$ G. Spedding, F. Brownand, and A. Fincham, "Turbulence, similarity scaling and vortex geometry in the wake of a towed sphere in a stablystratified fluid," J. Fluid Mech. 314, 53 (1996).

${ }^{4}$ M. Bonnier, O. Eiff, and P. Bonneton, "On the density structure of farwake vortices in a stratified fluid," Dyn. Atmos. Oceans 31, 117 (2000).

${ }^{5}$ P. Billant and J. Chomaz, "Experimental evidence for a zigzag instability of a vertical columnar vortex pair in a strongly stratified fluid," J. Fluid Mech. 418, 167 (2000).

${ }^{6} \mathrm{M}$. Beckers, R. Verzicco, H. Clercx, and G. van Heijst, "Dynamics of pancake-like vortices in a stratified fluid: Experiments, model and numerical simulations," J. Fluid Mech. 433, 1 (2001).

${ }^{7} \mathrm{D}$. Lilly, "Stratified turbulence and the mesoscale variability of the atmosphere," J. Atmos. Sci. 40, 749 (1983).

${ }^{8}$ D. Tritton, Physical Fluid Dynamics, 2nd ed. (Oxford University Press, Oxford, 1988).

${ }^{9}$ L. Armi, D. Hebert, N. Oakey, J. Price, P. Richardson, T. Rossby, and B. Ruddick, "The history and decay of a Mediterranean salt lens," Nature (London) 333, 649 (1988). 